\title{
A Decentralized Approach for Optimal Wholesale Cross-Border Trade Planning Using Multi-Agent Technology
}

\author{
Ping Wei, Student Member, IEEE, Yonghe Yan, Yixin Ni, Senior Member, IEEE, Jerome Yen, and \\ Felix F. Wu, Fellow, IEEE
}

\begin{abstract}
Over the past decade, power industry has been undergoing deregulations to introduce competitions among market participants. Once centralized decision making must now adapt to the new market structure. The optimal cross-border electricity trade planning is an important issue in interconnected power systems under transmission open access. In this paper a decentralized approach is suggested to solve the problem using multi-agent technology. In the new approach rational market participants make decisions based on their own benefits, in the meantime the minimum production and transmission cost of the whole system can be reached without a central coordination except necessary information exchange through media like the Internet. A relevant lemma has been proven. The approach is implemented via a multi-agent system using Java programming language. Computer tests on a 5-area test system show that the suggested new approach is effective and promising.
\end{abstract}

Index Terms-Cross-border trade plan, decentralized optimization, multi-agent technology, power market.

\section{INTRODUCTION}

$\mathbf{O}$ VER the past decade, electric power systems have been undergoing deregulations to introduce competitions among market participants, see [1], [2]. Transmission open access for suppliers and customers is one of the most prevailing unbundling services [3], [4]. It requires the owners of transmission systems to allow other wholesale entities to use transmission capacities to transport electrical energy without discrimination.

Usually interconnected power systems are geographically owned by several regional investors and the regional systems are connected together through tie-lines. The electricity trade inside a regional system can be handled locally. While the electricity trades among regional systems should pay for the usage of tie lines and the regional networks along the transaction paths [5]. It is clear that wholesale cross-border trades should be considered together with the transmission cost with tie line capacity limits included.

Manuscript received August 9, 2000; revised May 21, 2001. This research was supported by National Key Basic Research Special Fund (1998020308 and 1998020305), RGC Grant, HK SAR and CRCG Grant, and HKU.

P. Wei, Y. Ni, and F. F. Wu are with the Department of Electrical and Electronic Engineering, The University of Hong Kong, Hong Kong SAR, China.

Y. Yan is with the School of Computer Science, Telecommunication and Information System, Depaul University, Chicago, IL 60604.

$\mathrm{J}$. Yen is with the Department of Systems Engineering and Engineering Management, Chinese University of Hong Kong, Hong Kong SAR, China.

Publisher Item Identifier S 0885-8950(01)09441-X.
There are two basic approaches to handle the wholesale crossborder trade schedule. One is the centralized scheduling approach where the market operator is responsible to work out an optimal cross-border transmission schedule to satisfy the demands at the minimum production and transmission cost with consideration of system operation constraints and then allocates the transmission cost to individual transactions. This kind of centralized scheduling approach is widely used in the world now, say in California [6], England [7], et al., and various centralized optimization methods can be used for this task. Details of this topic can be found in [8] and [9]. As for the cost allocation task, [10] suggests a power flow based MW-mile method to allocate the cost. Reference [11] proposed a physical-DC-flowbased approach to allocate costs in which the transactions are explicitly expressed. However, very often market participants might doubt the fairness of the centrally announced results. Besides the central optimization has to repeat together with associated transmission cost allocation whenever a new transaction is added.

The other approach is the decentralized scheduling, which uses the invisible market hand, i.e., profit driving, to solve the problem by market participants themselves. The pioneering research was done by F. F. Wu, et al. [12]. They suggested a new paradigm, where economic decision is carried out by private multilateral trades among generators and consumers, while the function of reliability is coordinated through the PSO. Based on above idea, one implementation [13] uses the Bilateral Shapley Value to negotiate in multilateral trades via a multi-agent system. This approach avoids the centralized decision making of the market operator and is quite attractive to market participants. However, the optimal social welfare is not guaranteed.

In this paper, we are going to suggest a new decentralized method base on the rule of "first come, first serve" to implement the cross-border trade planning, which can encourage the market participants to compete for the system resources in advance. This point is demonstrated through the computer test results later. In the new decentralized method each regional system makes its own decision on cross-border trades, which is beneficial to itself without a central coordinating figure. However, it is proven in the paper that the total transmission cost minimized after such "selfish" decisions. The significant advantage of this approach lies in that it is based on each participant's rational behavior and any unfair central scheduling can be avoided. 
The suggested approach is successfully implemented with the help of Internet and related technologies, such as, World-wide Web (WWW) and Multi-agent Technology (MAT). Essentially, MAT is viewed as part of a wider area of Distributed Artificial Intelligence with each agent as an intelligent software agent [14]. In our MAT system, each regional system acts as an autonomous agent with the market information transmitted through Internet. So the agents are distributed, independent and intelligent soft "robots" for decision making. One more agent is assigned for central information handling. And the MAT system provides a flexible and effective platform for their interaction. Through this approach, the transparency and efficiency of market operation can be reached.

We shall first present the mathematical model of the problem in its centralized optimal format. Then our decentralized approach is introduced. The lemma that our approach is consistent to the centralized optimal result is proved thereafter. The suggested approach has been implemented using multi-agent technology. A 5-area test system is used to show the relevant calculation and the consistence of our method with central optimal approach. The computer test results show that the suggested new approach is effective and promising.

\section{Mathematical Model}

The basic assumptions used in our study are as follows:

a) The transmission price of each tie line is a constant and announced in \$ per unit power flow. The transmission limits of tie lines are known in per unit. For simplicity, the transmission loss is neglected (its cost can be included approximately into the tie line transmission price) and the power flow of each tie line is controllable.

b) An area is clarified as a supply, demand or transit area if its net injection power $P_{i}$ is greater than, less than or equal to zero. $P_{i, \max }$ is the net injection capacity of area $i$ when $P_{i}>0$. The net generation capacity $P_{i, \max }$ for a supply area and the load demand $P_{i, \text { load }}$ of a demand area are all known.

c) There are enough generation capacities to meet the load demands in the entire inter-connected system and the tie-line capacity is enough so that all the demands can be satisfied via proper schedule.

d) Although there is no central coordinator, all market information (including network structures, announced prices and available capacities, etc.) is made available on a continuously updated basis to all market participants through a central server. And each participant behaves rationally and pursues own interests.

For the wholesale cross-border trades scheduling problem, the math model for the centralized optimal decision can be formulated as follows:

$$
\begin{gathered}
\min \sum_{(i, j) \in A} c_{i j} \cdot f_{i j}+\sum_{i \in S}\left(t_{i} \cdot \sum_{j:(i, j) \in A} f_{i j}\right) \\
+\sum_{i \in D, T}\left(t_{i} \cdot \sum_{k:(k, i) \in A} f_{k i}\right)
\end{gathered}
$$

$$
\begin{aligned}
& \text { s.t. } \sum_{j:(i, j) \in A} f_{i j}-\sum_{k:(k, i) \in A} f_{k i}=P_{i} \quad \text { for } i=1,2, \ldots, n \\
& 0 \leq f_{i j} \leq f_{i j, \max } \quad \text { for all }(i, j) \in A \\
& 0 \leq P_{i} \leq P_{i, \max } \quad \text { for all } i \in S
\end{aligned}
$$

where

$f_{i j} \quad$ power flow on the tie-line from region $i$ to region $j$, and $f_{i j}>0$;

$f_{i j, \max } \quad$ capacity of the lie-line from region $i$ to region $j$;

$c_{i j} \quad$ price of per unit power flow for usage of tie-line $(i, j)$;

$t_{i} \quad$ price of per unit power flow for usage of network of area $i$;

$S, D, T$ denote supply, demand and transit area sets, respectively;

A entire tie line set with $\mathrm{m}$ directed tie-line flows; $n$ total number of areas.

The three terms of the objective function in (1) are the total costs for tie-line usage; the total fees for the usage of all supply area networks; and the total fees for the usage of other area networks respectively. It should be noticed that the generation cost is not included in the objective function for simplicity. There is no difficulty to include generation cost of each supply area into the problem. This is realized through introducing a fictitious "supply area-tie line" set with the tie line transmission price equal to the generation cost of the supply area. Then the original supply area becomes a "transit" area in the new system.

Now we start to introduce our decentralized approach based on the rule of "first come, first serve." We shall prove that after successive rational decisions of the cross-border trades by demand areas the global optimal solution of (1) can be reached.

For ease of description, we number supply areas from 1 to $n_{S}$, transmit areas from $n_{S}+1$ to $n_{S}+n_{T}$, and load areas from $n_{S}+n_{T}+1$ to $n_{S}+n_{T}+n_{D}=n$. The sequence of load numbers also represents the sequence of requests for power supply, or say the sequence of trade decision makings. Our decentralized optimal approach is completed in three steps as follows.

Step 1) Based on the definition in (1), we begin with a system without any cross-border transaction. Set tie line power flow vector initial value as zero, i.e., $F^{(0)}:=0$. Set area power vector initially as $e^{(0)}:=P^{(0)}$ with its $i$ th element $P_{i}^{(0)}$ equal to:

$\begin{cases}P_{i}^{(0)}=P_{i, \max } & i=1, \ldots, n_{S} \\ P_{i}^{(0)}=0 & i=n_{S}+1, \ldots, n_{S}+n_{T} \\ P_{i}^{(0)}=-P_{i, \text { load }} & i=n_{S}+n_{T}+1, \ldots, n_{S}+n_{T}+n_{D}\end{cases}$

i.e., for a supply area (node) $P_{i}^{(0)}$ is its available capacity, for a demand area (node) $P_{i}^{(0)}$ is $(-1)$ times its un-served demand.

Step 2) For $i=n_{S}+n_{T}+1$ (the first served demand area), go through the following sub-steps.

a) Determine the cheapest path $\psi_{i}$ to get one unit power from a supply area, say $s$.

b) Augment $\delta$-unit flow $(\delta>0)$ along path $\psi_{i}$ from area $s$ to area $i$, where 
$\delta:=\min (e(s),-e(i)$, available capacity along $\left.\psi_{i}\right)$.

c) Update $F, e(i)$ and $e(s)$ :

$$
F^{(n e w)}=F^{(o l d)}+\Delta F,
$$

where $\Delta F=\left(0, \ldots, 0, \Delta f_{\Psi_{i}}, 0, \ldots, 0\right)$ with the magnitude of $\Delta f_{\Psi_{i}}$ equal to $\delta, \Psi_{i}$ is the arc set of the path from $s$ to $i$

$$
\begin{aligned}
e^{(n e w)}(s) & =e^{(\text {old })}(s)-\delta \\
e^{(n e w)}(i) & =e^{(o l d)}(i)+\delta .
\end{aligned}
$$

d) Repeat steps $a$ ) to $c$ ) until $e(i)=0$, i.e., the corresponding demand is totally served.

Step 3) Set $i:=i+1$, and conduct all sub-steps a) $-\mathrm{d}$ ) in step 2). Repeat step 3) until $i=n_{S}+n_{T}+n_{D}=n$. The final optimal cross-border schedule can be known from the tie line flow vector $F$.

Lemma: The result from the decentralized approach above is the same as that from the centralized approach defined in (1).

Proof: The lemma can be proven by the inductive method as follows.

a) When $j=1$, if the system has only one demand at node $d 1$ and it can be served by a single cheapest path $\Psi_{d 1}$ from a source node $s 1$ with system constraints satisfied, i.e.,

$P_{l o a d}^{(1)}=\min \left(e(s 1),-e(d 1)\right.$, available capacity along $\left.\psi_{d 1}\right)$.

It is clear that the corresponding tie line flow vector $F^{(1)}$ is also the global optimal solution of (1) for $P_{\text {load }}^{(1)}$. This is because that there are no other demands in the system and there are no other paths that are cheaper than the selected path (otherwise the selected path wouldn't be the cheapest path, which conflicts with the known condition). Therefore we have:

$$
F^{(1)}=F_{(1)}^{*}
$$

where $F_{(1)}^{*}$ is the corresponding centralized optimal solution [see (1)] when the system has unique demand 1.

If the demand at node $d 1$ can't be served by only one cheapest path because of system constraints, we can split the demand properly into two demands with one of them meeting the requirement as the first demand. So for the first demand we can always have $F^{(1)}=F_{(1)}^{*}$ and the corresponding transmission cost $C\left(F^{(1)}\right)=C\left(F_{(1)}^{*}\right)$.

b) Suppose that when $j=k$, there is

$$
F^{(k)}=F_{(k)}^{*}
$$

where $F_{(k)}^{*}$ is the tie line flow vector corresponding to the minimal cost from centralized optimal approach [see (1)] when demands 1 through $k$ are served; $F^{(k)}$ denotes the resultant tie line flow vector after successive rational decisions by demands 1 through $k$ individually using the cheapest path approach with system constraints included. Based on this assumption, we shall use "reduction to absurdity" to prove in the next step when $j=k+1$, the rational decision by the $(k+1)$ th demand will lead to $F^{(k+1)}=F_{(k+1)}^{*}$.

c) When $j=k+1$, we search a cheapest path $\Psi_{d m}$ from available source areas to the $(k+1)$ th demand area (denoted as node $d m$ ). If we consider the flow on the found cheapest path $\Psi_{d m}$ as the system demand increment $\Delta P_{\text {load }}^{(k+1)}$ :

$$
\begin{gathered}
\Delta P_{\text {load }}^{(k+1)}=\min (e(s m),-e(d m), \text { available capacity } \\
\text { along } \left.\psi_{d m}\right)
\end{gathered}
$$

where $s m$ is the source area number of the cheapest path $\Psi_{d m}$. If the corresponding tie line flow vector increment is $\Delta F^{(k+1)}$, we have:

$$
F^{(k+1)}=F^{(k)}+\Delta F^{(k+1)}=F_{(k)}^{*}+\Delta F^{(k+1)} .
$$

Suppose the resultant $F^{(k+1)}$ is not the minimal cost flow solution from the centralized optimal approach $F_{(k+1)}^{*}$ for the load vector $P_{\text {load }}^{(k+1)}$. Then the transmission cost $C\left(F^{(k+1)}\right)$ must be larger than $C\left(F_{(k+1)}^{*}\right)$, i.e.,

$$
C\left(F^{(k+1)}\right)>C\left(F_{(k+1)}^{*}\right) .
$$

(Here we assume the global optimal solution is unique.)

Then in order to serve $\Delta P_{\text {load }}^{(k+1)}$, we can constitute another tie line flow increment vector as

$$
\Delta F_{(k+1)}^{\prime}=F_{(k+1)}^{*}-F_{(k)}^{*} .
$$

Due to the linearity of the cost function, substituting (6) into (5) and considering (4), we can rewrite (5) as:

$$
\begin{aligned}
C\left(F_{(k)}^{*}\right)+C\left(\Delta F^{(k+1)}\right) & >C\left(F_{(k)}^{*}\right)+C\left(\Delta F_{(k+1)}^{\prime}\right) \\
\therefore \quad C\left(\Delta F^{(k+1)}\right) & >C\left(\Delta F_{(k+1)}^{\prime}\right) .
\end{aligned}
$$

It is obvious that (7) contradicts with the condition that $\Delta F^{(k+1)}$ is the cheapest path for $\Delta P_{\text {load }}^{(k+1)}$. Therefore (5) is not true and $F^{(k+1)}$ must have minimum transmission cost corresponding to $F_{(k+1)}^{*}$ for the load vector $P_{\text {load }}^{(k+1)}$.

d) According to the proof, we know that when all the $n_{D}$ demands are served one by one based on the cheapest-path search at $j=k_{\text {end }}$, the corresponding tie line flow vector $F^{\left(k_{e n d}\right)}$ will also be the global optimal cross-border trade solution of (1).

End of Proof.

The advantages of the new approach are apparent:

a) There is no need for a central coordinator. Each demand area searches for its cheapest path to satisfy its own need.

b) Every demand area is satisfied with its choice based on the available cheapest path and does not need to worry about the bias from central processing.

c) The minimal total transmission cost can still be guaranteed at the end with system constraints satisfied.

d) Since the method is based on the rule of "first come, first serve," when a new trade is added, previous trade schedules will not change. This is extremely attractive as compared with centralized optimization approaches.

e) The new approach does not need transmission cost allocation calculation since it can obtain transmission cost of each trade during the process. 


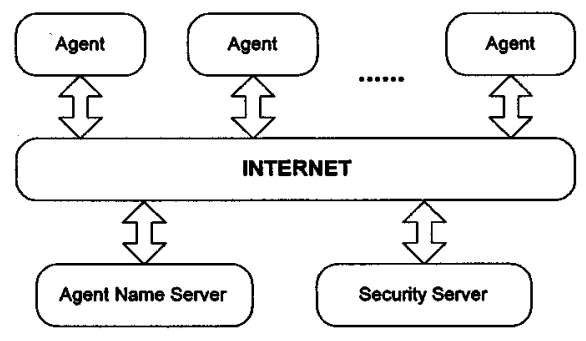

Fig. 1. Infrastructure of MASCBTP.

f) The area power generation cost can also be included easily by introducing a fictitious "supply area-tie line" set as mentioned before.

\section{IMPLEMENTATION}

The new suggested method has been implemented on the Internet using multi-agent technology. The developed system is called MASCBTP (Multi-agent System for Cross-border Trade Planning). Fig. 1 shows the infrastructure of MASCBTP. Agent communication is done via the Internet. The agent name server manages the agent registration service and other administration tasks. The security server is responsible for approving crossborder trade requests from the participating agents according to system constraints and the rule of "first come first serve," and it is also responsible for updating the information after each approval. The two servers can be merged into one. In view of the suggested decentralized approach, the security server is not necessary since all the decisions can be rationally made and announced by the individual agents themselves. But for the practical implementation, we suggest a central server to guarantee the security of the system and to improve the speed of the processing and system management.

Each regional network is assumed to be a rational agent and the communication channels are also assumed to be perfect. Based on the information updated by the security server, each demand agent installed with similar software searches the cheapest path to meet its own demand. A generic label-correcting algorithm is used in MASCBTP to compute the cheapest path. The algorithm is a general procedure for successively updating the cost labels until they satisfy the cheapest path optimality condition. The details of the algorithm can be found in [15].

After an agent has made its decision, it will send its request to the security server. If the security server approves the request according to the rule of "first come first serve," it will announce the information via the Internet. Then under the new environment, other agents will begin a new round of calculation and compete for the cheapest path to supply their un-served demands. The process will continue till all the demands are satisfied.

\section{Computer Test Results}

A 5-area test system [16] (see Fig. 2) is used for computer test and to show how our method works based on multi-agent technology.

In Fig. 2, each bigger circle represents a regional network connected by tie lines to other networks. The number inside a circle represents the assumed transmission cost of per unit flow

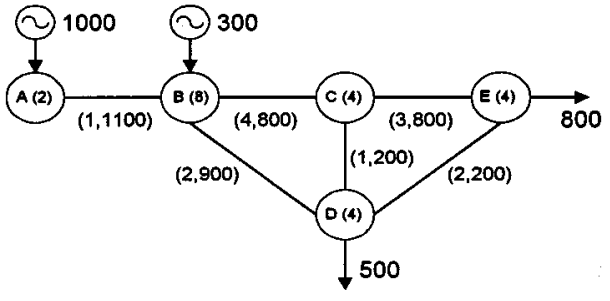

Fig. 2. The test system schematic diagram.

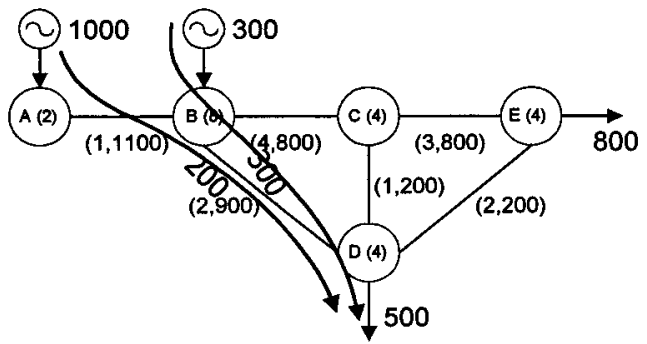

(a) First request from agent $\mathrm{D}$

Cost of the trade B-D: $(8+2+4) \times 300=4200$

Cost of the trade A-B-D: $(2+1+8+2+4) \times 200=3400$

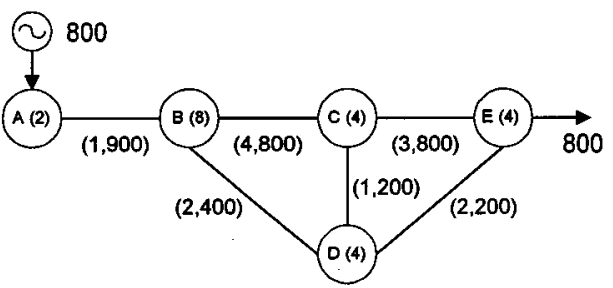

(b) Information updated after the request from D is approved

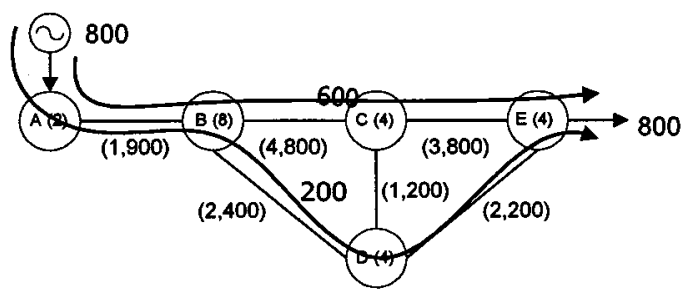

(c) Second request from agent $\mathrm{E}$

Cost of the trade A-B-D-E: $(2+1+8+2+4+2+4) \times 200=4600$

Cost of the trade A-B-C-E: $(2+1+8+4+4+3+4) \times 600=15600$

Fig. 3. Illustration of calculation process of case 1 .

for transit through the regional systems. Each tie line has two parameters put in a parenthesis. The first number represents the transmission cost for per unit flow and the second number the transmission capacity of the tie line. The number by the side of a generator (or a demand) means the available generation capability (or the amount of load demand).

For simplicity generation cost is assumed to be the same and neglected in optimization. Two cases are studied: in case 1 agent $D$ sends its request first; and in case 2 agent $\mathrm{E}$ does it first. The results are presented below.

Case 1: The request from agent $\mathrm{D}$ is sent first.

1st Round: The cheapest path for agent $\mathrm{D}$ is the path $\mathrm{B}-\mathrm{D}$ with per unit flow cost equal to $(8+2+4=14)$. Because of the generation capacity limit, agent B can only supply 300 -unit power. Agent $\mathrm{D}$ has to find another cheapest path for the rest of demand from A through $\mathrm{B}$ to $\mathrm{D}$ with the transmission cost rate 17, as illustrated by Fig. 3(a). After the cheapest cost calculation, agent $\mathrm{D}$ sends its request for these two trades to the 


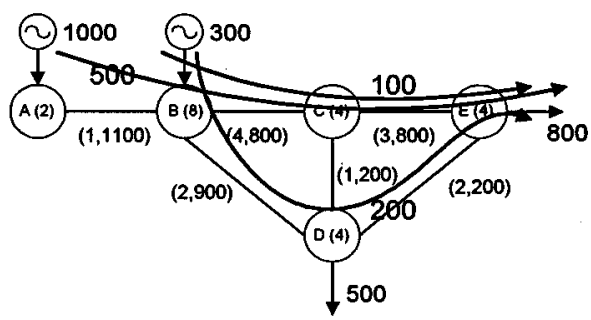

(a) First request from agent $\mathrm{E}$

Cost of the trade B-D-E: $(8+2+4+2+4) \times 200=4000$

Cost of the trade B-C-E: $(8+4+4+3+4) \times 100=2300$

Cost of the trade A-B-C-E: $(2+1+8+4+4+3+4) \times 500=13000$

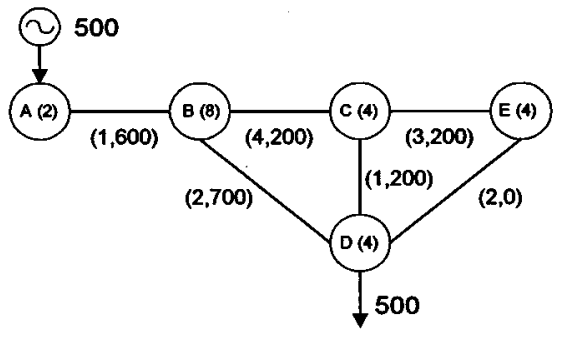

(b) Information updated after the request from $\mathrm{E}$ is approved

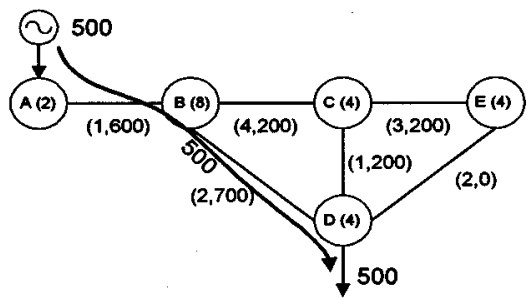

(c) Second request from agent $D$

Cost of the trade A-B-D: $(2+1+8+2+4) \times 500=8500$

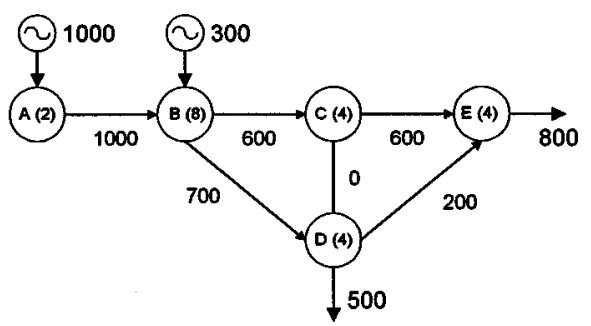

(d) Final solution of the system flow

Fig. 4. Illustration of calculation process of case 2 .

security server. At the same time, agent $\mathrm{E}$ is also calculating the cheapest trades as shown in Fig. 4(a), and sends the request to the security server as well. However, the request from agent D arrives first and gets approved first. The information is then updated as shown in Fig. 3(b).

2nd Round: Based on the updated information, agent $\mathrm{E}$ finds the cheapest path is A-B-D-E with the cost rate 23. Due to the transmission capacity limit of tie line D-E, only 200-unit power can be supplied along the path. Then agent $\mathrm{E}$ tries to find another cheapest path for the rest of demand. It is A-B-C-E with the cost rate 26, as shown in Fig. 3(c). The security server will approve the request from $\mathrm{E}$ for the two trades.

At the end of processing, all the demands are met. And every load area obtains the available cheapest paths for their trades under existing circumstances. According to Fig. 3(a) and (b), we know the transmission price for agent $\mathrm{D}$ is $4200+3400=$
7600 and for agent $\mathrm{E}$ is $4600+15600=20200$. The total transmission cost is $7600+20200=27800$.

Case 2: The request of agent $\mathrm{E}$ is sent first.

1st Round: Based on the initial information shown in Fig. 2, agent $E$ calculates the cheapest path to meet its demands. It finds the path B-D-E with the cost rate 20. Due to the transmission capacity limit of tie line D-E, this trade can only be 200-unit power. Then the second cheapest path is found to be B-C-E with the cost rate 23. However due to the limitation of generation capacity, the magnitude of the trade is limited to 100-unit power. The last cheapest path is A-B-C-E with the cost rate 26 and the demand of agent $\mathrm{E}$ is finally satisfied. The request for the three trades [see Fig. 4(a)] is sent to security server. In the mean time, agent $\mathrm{D}$ also calculates its cheapest trade plan as shown in Fig. 3(a), but it sends out a little later. So the security server approves the request from agent $\mathrm{E}$ first. And the updated information is shown in Fig. 4(b).

2nd Round: Based on the updated information, agent $\mathrm{D}$ calculates the cheapest path again and sends the request to the security server. The cheapest path is from A through B to D with the cost rate 17 shown in Fig. 4(c). The trade is 500-unit power.

In case 2 agent $\mathrm{D}$ should pay 8500 for cross-border trades which is more expensive than it paid in case 1 , however agent E will only pay $4000+2300+13000=19300$ for its trades, which is cheaper than 20200 it paid in case 1. It is easy to prove that the winner in the competition for the cheapest path is the one who gets most benefits [17], which is consistent with the rule of "first come, first serve." It is clear that under such scheme, each market participant is encouraged to corner system resources for its own benefit in advance. The earlier it makes decision, the more benefits it can obtain.

A summary of the trade costs is listed in Table I. In case 1, agents D and E paid 7600 and 20200 respectively. In case 2, agents D and E paid 8500 and 19300 respectively. The total transmission cost is 27800 for both cases, which is also equal to the result of centralized optimization defined in (1). The resultant power flow for cases 1 and 2 and the centralized optimal approach are the same, which is shown in Fig. 4(d).

It should be pointed out again that using the decentralized approach, no further transmission cost allocation is required since it is implemented in parallel with the decentralized process, which makes the method more attractive than the centralized approaches.

Due to the same final power flow shown in Fig. 4(d), each participant receives same amount of money in the different cases (see the last column of Table I).

\section{CONCLUSION}

In this paper, we proposed a decentralized approach to make cross-border trade planning using multi-agent technology under transmission open access, in which each market participant making decision independently to seek its own profit, i.e., to compete for the cheapest path. The advantage of this approach lies in that no centralized control is required to guarantee the autonomous behavior, but minimum transmission cost of the whole system can be achieved finally, which is proven in the paper. Furthermore, when a new trade is added, it will use 
TABLE I

Cost Allocation (IN MONEy UnITS)

\begin{tabular}{c|c|c|c|c}
\hline \multicolumn{2}{c|}{ Cost flow } & $\begin{array}{c}\text { Payment } \\
\text { in Case 1 }\end{array}$ & $\begin{array}{c}\text { Payment } \\
\text { in Case 2 }\end{array}$ & $\begin{array}{c}\text { Income from } \\
\text { service }\end{array}$ \\
\hline \multirow{4}{*}{$\begin{array}{c}\text { Area } \\
\text { Network }\end{array}$} & A & 0 & 0 & 2000 \\
\cline { 2 - 5 } & B & 0 & 0 & 10400 \\
\cline { 2 - 5 } & C & 0 & 0 & 2400 \\
\cline { 2 - 5 } & D & 7600 & 8500 & 2800 \\
\cline { 2 - 5 } & E & 20200 & 19300 & 3200 \\
\hline \multirow{5}{*}{ Tie line } & A-B & 0 & 0 & 1000 \\
\cline { 2 - 5 } & B-C & 0 & 0 & 2400 \\
\cline { 2 - 5 } & C-E & 0 & 0 & 1800 \\
\cline { 2 - 5 } & B-D & 0 & 0 & 1400 \\
\cline { 2 - 5 } & C-D & 0 & 0 & 0 \\
\cline { 2 - 5 } & E-D & 0 & 0 & 400 \\
\hline \multicolumn{2}{c|}{ Total } & 27800 & 27800 & 27800 \\
\hline
\end{tabular}

remaining transmission resources, therefore previous trade schedules will not change, and the system-wide re-coordination calculations, usually required in centralized approach through OPF, are avoided.

The approach is successfully implemented through a multiagent system called MASCBTP using Java programming language. Computer tests on a 5-area system show that the suggested approach is effective and promising.

\section{REFERENCES}

[1] H.-p. Chao and H. G. Huntington, Designing Competitive Electricity Markets: Kluwer Academic Publishers, 1998.

[2] M. Ilic, F. Galiana, and L. Fink, Power Systems Restructuring: Engineering and Economics: Kluwer Academic Publishers, 1998.

[3] H. H. Happ, "Cost of wheeling methodologies," IEEE Trans. Power Systems, vol. 9, no. 1, Feb. 1994.

[4] R. D. Tabors, "Transmission system management and pricing: New paradigms and international comparisons," IEEE Trans. Power Systems, vol. 9, no. 1, Feb. 1994.

[5] J. W. Bialek, "Tracing-based unifying framework for transmission pricing of cross-border trades in Europe," in Proceedings of DRPT'2000, London, Apr. 2000

[6] P. R. Gribik, G. A. Angelidis, and R. R. Kovacs, "Transmission access and pricing with multiple separate energy forward markets," IEEE Trans. Power Systems, vol. 14, no. 3, Aug. 1999.

[7] R. Green, "Transmission pricing in England and Wales," Utilities Policy, vol. 6, no. 3, Sept. 1997.

[8] IEEE Tutorial Course, "Optimal power flow: Solution techniques, requirements, and challenges," IEEE Power Engineering Society, 1996.

[9] F. C. Schweppe, M. C. Caramanis, R. D. Tabors, and R. E. Bohn, Spot Pricing of Electricity: Kluwer Academic Publishers, 1988

[10] D. Shirmohammadi, P. R. Gribik, and E. T. K. Law et al., "Evaluation of transmission network capacity use for wheeling transactions," IEEE Trans. Power Systems, vol. 4, no. 4, Oct. 1989.
[11] G. Gross and S. Tao, "A physical-flow-based approach to allocating transmission losses in a transaction framework," IEEE Trans. Power Systems, vol. 15, no. 2, May 2000.

[12] F. F. Wu and P. Varaiya, "Coordinated multilateral trades for electric power networks: Theory and implementation," International Journal of Electrical Power \& Energy Systems, vol. 21, no. 2, pp. 75-102, Feb. 1999.

[13] C. S. K. Yeung, A. S. Y. Poon, and F. F. Wu, "Game theoretical multiagent modeling of coalition formation for multilateral trades," IEEE Trans. Power Systems, vol. 14, no. 3, Aug. 1999.

[14] N. R. Jennings, Foundations of Distributed Artificial Intelligence. New York, NY: Wiley, 1996.

[15] R. K. Ahuja, T. L. Magnanti, and J. B. Orlin, Network Flows: Theory, Algorithms, and Applications. Englewood Cliffs, NJ: Prentice-Hall, 1993.

[16] "International exchanges of electricity-Rules proposed by the European Transmission System Operators,", 14.1.1999.

[17] Y. H. Yan, "A multi-agent based approach to transmission cost allocation," Doctoral thesis, The University of Hong Kong, 2000.

Ping Wei received the M.Eng. degree in electrical engineering from Southeast University, China. She is now a Ph.D. student, Dept. of EEE, the University of Hong Kong. Her research interests include power system operation and power market.

Yonghe Yan received the $\mathrm{Ph}$.D. degree in computer science from the University of Hong Kong in 2000. He joined Depaul University as an assistant professor. His research interests are E-commerce, AI and intelligent agents, distributed and collaborative computing.

Yixin Ni received the B.Eng., M.Eng., and Dr.Eng. degrees all in electrical engineering from Tsinghua University, China. She was former professor and director of National Power System Lab, Tsinghua University and now with the University of Hong Kong. Her research interests are power system stability and control, FACTS, AI tech. applications in power systems and power markets.

Jerome Yen received the $\mathrm{Ph} . \mathrm{D}$. degree in systems engineering and management information systems from the University of Arizona in 1992. He is now an associate professor in Chinese University of Hong Kong. His major research interests include next generation Internet, E-commerce, digital library and information markets.

Felix F. Wu received the Ph.D. degree from University of California at Berkeley (UCB). He is now a chair professor of electrical engineering and vice president of the University of Hong Kong. Prior to that he was a professor and vice-chair of Dept. of EECS, UCB. His research interests are electric energy industry restructuring, power system investment planning, design of modern control centres, distribution automation, distributed processing, etc. 